\title{
Keratinocytes and Activation of TREM-1 Pathway in Cutaneous Leishmaniasis Lesions
}

\author{
Sara Nunes ${ }^{1,2} \mathbb{D}$, Mariana Rosa Ampuero ${ }^{1,2}$, Ícaro Bonyek-Silva 1,2 ${ }^{\mathbb{D}}$, Reinan Lima ${ }^{1,2}$, Filipe Rocha Lima ${ }^{1,2} \mathbb{D}$, \\ Sérgio Marcos Arruda 1,2, Ricardo Khouri 1,2 ${ }^{(D)}$, Pablo Rafael Silveira Oliveira $2 \mathbb{D}$, Aldina Barral 1,2,3, \\ Viviane Sampaio Boaventura ${ }^{1,2}$, Cláudia Ida Brodskyn ${ }^{1,2,3}$ and Natalia Machado Tavares ${ }^{1,2, *}$ \\ 1 LaIPHE, Oswaldo Cruz Foundation, Gonçalo Moniz Institute, FIOCRUZ, Salvador 40296-710, Bahia, Brazil; \\ sara_nunes2@hotmail.com (S.N.); mariana.ampuero@hotmail.com (M.R.A.); icaro.bonyek@gmail.com (Í.B.-S.); \\ reinanlimadeus@hotmail.com (R.L.); rfilipelima@gmail.com (F.R.L.); sergio.arruda@fiocruz.br (S.M.A.); \\ ricardo_khouri@hotmail.com (R.K.); aldina.barral@fiocruz.br (A.B.); viviane.boaventura@fiocruz.br (V.S.B.); \\ claudia.brodskyn@fiocruz.br (C.I.B.) \\ 2 School of Medicine and Institute of Biology (IBIO), Federal University of Bahia, \\ Salvador 40110-100, Bahia, Brazil; pablorafael_ssa@hotmail.com \\ 3 Instituto Nacional de Ciência e Tecnologia (INCT) iii-Instituto de investigação em Imunologia, \\ São Paulo 05401-350, São Paulo, Brazil \\ * Correspondence: natalia.tavares@fiocruz.br
}

check for updates

Citation: Nunes, S.; Ampuero, M.R.; Bonyek-Silva, Í; Lima, R.; Lima, F.R.; Arruda, S.M.; Khouri, R.; Oliveira, P.R.S.; Barral, A.; Boaventura, V.S.; et al. Keratinocytes and Activation of TREM-1 Pathway in Cutaneous Leishmaniasis Lesions. Microbiol. Res. 2021, 12, 765-778. https://doi.org/ 10.3390/microbiolres12040056

Academic Editor: Sofia Casares

Received: 26 June 2021

Accepted: 26 August 2021

Published: 7 October 2021

Publisher's Note: MDPI stays neutral with regard to jurisdictional claims in published maps and institutional affiliations.

Copyright: (c) 2021 by the authors. Licensee MDPI, Basel, Switzerland. This article is an open access article distributed under the terms and conditions of the Creative Commons Attribution (CC BY) license (https:/ / creativecommons.org/licenses/by/ $4.0 /)$.
Abstract: Triggering Receptor Expressed on Myeloid Cells 1 (TREM-1) amplifies the immune response, operating synergistically with Toll-Like Receptors (TLRs) in the production of inflammatory mediators. TREM-1 signaling depends on the adapter protein DAP12, which results in the activation of $\mathrm{NFkB}$, the expression of inflammatory genes, and the release of antimicrobial peptides, such as Beta-defensin 2. We evaluated the activation of the TREM-1 signaling pathways in Cutaneous Leishmaniasis (CL) caused by Leishmania braziliensis and linage human keratinocytes exposed to these parasites since the host immune response against Leishmania plays a critical role in promoting parasite killing but also participates in inflammation and tissue damage. We analyzed publicly available transcriptome data from the lesions of CL patients. In the CL biopsies, we found increased expression of the molecules involved in the TREM-1 pathway. We then validated these findings with RT-qPCR and immunohistochemistry in newly obtained biopsies. Surprisingly, we found a strong labeling of TREM-1 in keratinocytes, prompting the hypothesis that increased TREM-1 activation may be the result of tissue damage. However, increased TREM-1 expression was only seen in human lineage keratinocytes following parasite stimulation. Moreover, no up-regulation of TREM-1 expression was observed in the skin lesions caused by other non-infectious inflammatory diseases. Together, these findings indicate that L. braziliensis $(\mathrm{Lb})$ induces the expression of the TREM-1 receptor in tissue keratinocytes regardless of tissue damage, suggesting that non-immune skin cells may play a role in the inflammatory response of CL.

Keywords: Leishmania; inflammation; TREM-1; keratinocytes

\section{Introduction}

Leishmaniasis is a complex of neglected tropical diseases caused by a protozoan parasite of the genus Leishmania. Among them, cutaneous leishmaniasis (CL) is the most frequent form of the disease. The immune response in cutaneous leishmaniasis caused by Leishmania braziliensis $(L b)$ is considered highly inflammatory, which is crucial for parasite killing but leads to tissue damage [1-4]. CL lesions are chronic skin ulcers with raised edges and a necrotic center. Its development depends on the Leishmania species in addition to a combination of factors associated with the host immune response, which defines different clinical outcomes. Several studies have suggested that the severity of CL is more associated with an exacerbated inflammatory response than a consequence of parasite burden. Chronic and exacerbated inflammation of CL has been associated with the high 
production of pro-inflammatory cytokines and increased expression of pattern recognition receptors (PRRs), such as TREM-1, which amplify the innate immune response against pathogens $[1,3,5,6]$.

TREM-1, the triggering receptor expressed in myeloid cells, is an activating receptor present in neutrophils, monocytes, and macrophages [7]. It is activated during inflammatory and infectious conditions that involve the release of high levels of pro-inflammatory cytokines and chemokines. TREM-1 signals through the DAP12 adapter protein (DNAX activation protein of $12 \mathrm{kDa}$ ), and it can be cleaved into its soluble form (sTREM-1), which is crucial to modulate cell signaling [8-10]. TREM-1 expression has been associated with excessive inflammation and tissue damage rather than pathogen clearance [6]. In this context, Carneiro and collaborators (2016) [11] have already identified the activation of the TREM-1 pathway in cells from healthy individuals who are high producers of IFN- $\gamma$ in response to L. braziliensis stimulation in vitro. More recently, our group identified the up-regulation of the TREM-1 pathway and its gene as a potential target for microRNAs in lesions caused by L. braziliensis associated with a good response to treatment and CL prognosis [12].

Studies with in vitro and in vivo approaches have investigated the TREM-1 pathway in leishmaniasis. However, few aspects of TREM-1 expression have been described in the context of human CL caused by L. braziliensis, especially with respect to the keratinocytes in the lesions. It has already been demonstrated that human neutrophils infected in vitro with L. infantum upregulate the expression of TREM-1, DAP-12, and IL-8. In addition, increased serum levels of the soluble form of TREM-1 (sTREM-1) were associated with the severity of visceral leishmaniasis (VL) [13]. Neutrophil recruitment to CL lesions has also been associated with TREM-1 in experimental models. Mice deficient for TREM-1 develop smaller CL lesions due to a reduced neutrophil infiltrate after L. major infection without altering the capacity to control the parasite. These studies suggest that TREM-1 could be a promising target for controlling exacerbated inflammation without interfering in parasite killing [6].

Regardless of the species, Leishmania infection begins in the skin, and it is crucial to understand the mechanisms involved in the early moments of lesion development. This will allow the development of new strategies to control exacerbated inflammation and tissue damage $[14,15]$. Keratinocytes are the major cell type found in the epidermis, the outermost layer of the skin [16]. They have been extensively studied in different skin lesions, but few studies have evaluated the interaction between keratinocytes and Leishmania spp. [5]. It is known that despite their phagocytic capacity, the interaction between Leishmania and keratinocytes does not result in significant amounts of internalized parasites [14,17]. In addition, their ability to proliferate inside the keratinocytes is compromised [14]. Although these findings do not suggest a close contact between Leishmania and keratinocytes, their interaction is considered immunomodulatory. The exposure of primary keratinocytes to $L$. major or L. infantum induced different responses in each case. A significant production of pro-inflammatory cytokines was observed in keratinocytes cultured with L. infantum compared to those cultured with L. major [14]. These data suggest that a non-immune cell type can influence the inflammatory response during Leishmania infection and, consequently, the disease outcome. However, there is a lack of knowledge regarding the involvement of the TREM-1 pathway and keratinocytes during L. braziliensis infection, the most prevalent species in Brazil.

In the present study, we sought to investigate the expression of the TREM-1 pathway in human CL lesions caused by L. braziliensis and to further localize its expression in the tissue of newly obtained biopsies. We found that the TREM-1 pathway is broadly activated in CL at both the gene and protein levels, mostly in the epidermis. The same results were found in the human adult keratinocyte cell line (Hacat) in vitro after L. braziliensis exposure, leading us to evaluate other inflammatory skin diseases. Taken together, this is the first study to localize TREM-1 in the lesions of humans caused by L. braziliensis. Furthermore, 
non-infectious inflammatory skin diseases do not modulate TREM-1 expression, suggesting that $L$. braziliensis induces the TREM-1 pathway regardless of tissue damage.

\section{Materials and Methods}

\subsection{Transcriptome and Pathway Analysis}

The Gene Expression Omnibus (GEO) (https://www.ncbi.nlm.nih.gov/geo/, accessed on 10 May 2017) repository was used to find transcriptome datasets related to "human biopsies", "Leishmania braziliensis" and "healthy control". Two sets of data that evaluated biopsies from patients with CL compared to healthy controls were found registered under the codes GSE55664 (Illumina HT12 v4 platform) and GSE63931(Agilent Sure Print GE Human G3v2 platform), which were published by Novais and collaborators [18] and Oliveira and collaborators [19], respectively. The former dataset compared 25 CL lesions (8 early and 17 late infections) with 10 healthy skin samples. The later dataset evaluated $8 \mathrm{CL}$ lesions (recently treated) and 8 healthy skin samples. Ingenuity Pathway Analysis software (IPA Tool; Ingenuity Systems; http:/ / www.ingenuity.com, accessed on 20 October 2017) was used to evaluate the data and to find the canonical pathways related with a cutaneous leishmaniasis.

The public GEO repository was later used to search for other transcriptome datasets for non-infectious inflammatory skin diseases: psoriasis (GSE53431) and systemic lupus erythematous (SLE) (GSE72535). In the psoriasis data set, 12 CL samples were compared to 12 healthy skin samples. Regarding the lupus transcriptome, nine CL samples were compared to nine control skins.

\subsection{Analysis of the TREM-1 Signaling Pathways}

Expression data were analyzed using the Multi Experiment Viewer (MeV) (www.tm4 .org/mev.html, accessed on 12 May 2017). Differentially expressed genes (DEGs) were determined based on the differences of variances between the two groups of samples $\left(\log _{2}\right)$. For the hierarchical cluster analysis, the distance between the samples was determined by the Euclidean distance and the average link (average-linkage). Heatmaps were used to represent the expression of the transcript probes through signal strength. Principal component (PCA) analysis grouped samples based on the expression of genes from the TREM-1 pathway, where PCA1 and PCA2 corresponded to more than $50 \%$ of the data variability. Volcano plots highlights the differences in the expression of the transcripts in the CL samples compared to the healthy control skin samples (Fold change (FC) $\geq 2.0$ ), with a Benjamini-Hoshberg False Discovery Rate (FDR)-adjusted $p$-value of $<0.05$ as a criterion to identify the DEGs.

\subsection{Patients and Ethics Statement}

The samples were obtained from 12 active lesions from CL patients from Jiquiriça, Bahia, Brazil. The diagnostic criteria were based on clinical signs of $C L$, histological characteristics, and positive response for late hypersensitivity (DTH). Biopsies were collected at the border of the lesions using a 4-mm punch before treatment. Additionally, seven control samples were taken from volunteers who were living in a non-endemic area without a history of leishmaniasis. The study was conducted according to the principles of the Declaration of Helsinki and under local ethical guidelines. This study was approved by the Ethical Committee of the Gonçalo Moniz Institute (Salvador, Bahia, Brazil—protocol number CAAE 42928215.9.0000.0040, approved in 4 July 2015). Informed consent was obtained from all patients.

\subsection{Cell Culture and Infection}

$\mathrm{HaCaT}$ cells (Lineage human keratinocytes) were cultured in $5 \% \mathrm{CO} 2$ at $37^{\circ} \mathrm{C}$ in regular Dulbecco's Modified Eagle's Medium (DMEM-Gibco), supplemented with 10\% fetal bovine serum and $2 \mathrm{mM}$ L-glutamine, $100 \mathrm{U} / \mathrm{mL}$ penicillin, and $100 \mu \mathrm{L} / \mathrm{mL}$ streptomycin 
(Invitrogen, San Francisco, CA, USA). The cells were seeded at a density of $5 \times 10^{5}$ cells, plated in 24-well plates, and cultured with for 5 days (70-80\% confluency).

Leishmania braziliensis MHOM/BR/01/BA788 were expanded in Schneider medium (Sigma) supplemented with $20 \%$ of fetal bovine serum (FBS) and 1\% PSG (2 mM Lglutamine, $100 \mathrm{U} / \mathrm{mL}$ penicillin and $100 \mu \mathrm{L} / \mathrm{mL}$ streptomycin). After 5 days of culture, metacyclic promastigotes in the stationary phase were used. The HaCat cells were exposed to Leishmania at a 5:1 ratio (parasite: cell) for $4 \mathrm{~h}$. In addition, some cells were subjected to mechanical lesions (wound healing) performed with a $200 \mu \mathrm{L}$ tip on the confluent monolayer in order to mimic lesions in these cells and to subsequently evaluate the expression of the culture. The cultures were washed with saline and were then placed in $700 \mu \mathrm{L}$ Trizol (Life technologies, Carlsbad, CA, USA) for RNA extraction (two independent experiments were performed in triplicate and quadruplicate).

\subsection{Quantitative Real Time Polymerase Chain Reaction (RT- $q P C R$ )}

Total RNA was extracted using the Qiagen RNeasy Mini Kit (Qiagen, Hilden, Germany) according to the manufacturer's protocols. The cDNA was generated using the SuperScript ${ }^{\mathrm{TM}}$ II Reverse Transcriptase Kit (Invitrogen, San Francisco, CA, USA), following the manufacturer's instructions. Gene expression was evaluated in duplicate on ABI 7500 real-time PCR equipment (Applied Biosystems, Foster City, CA, USA) following the manufacturer's instructions. The relative expression was calculated using the comparative limit cycle $(\mathrm{Ct})$ and was compared to the control (fold change) in addition to normalizing the reaction with the constitutive gene $\beta$-actin. The sequences of the primers are described in Table 1.

Table 1. Oligonucleotide sequences for primers used in the assay.

\begin{tabular}{cc}
\hline Gene & Primer Sequence \\
\hline \multirow{2}{*}{ TREM1 } & GAACTCCGAGCTGCAACTAAA (F) \\
TCTAGCGT GTAGTCACATTTCAC (R) \\
\multirow{2}{*}{ TYROBP } & ACTGAGACCGAGTCGCCTTAT \\
& (F) ATACGGCCTCTGTGTGTTGAG $(\mathrm{R})$ \\
\hline \multirow{2}{*}{ TLR2 } & CCTACTGGGTGGAGAACCTTAT (F) \\
& CAGGAATGAAGTCCCGCTTATG (R) \\
\hline \multirow{2}{*}{ DEFB4A } & CGC CTA TAC CAC CAA AAA CAC (F) \\
& TCC TGG TGA AGC TCC CA (R) \\
\hline \multirow{2}{*}{ ACTB } & CCT TGC ACA TGC CGG AG (F) \\
& ACA GAG CCT CGC CTT TG (R) \\
\hline
\end{tabular}

\subsection{Immunohistochemistry}

Immunohistochemistry was performed to evaluate the expression of TREM1, Def $\beta 2$, DAP12, and TLR2, following the manufacturer's instructions. Briefly, skin biopsies (six healthy skin samples and six CL lesions) were fixed with 10\% formalin, dehydrated, and embedded in paraffin. Then, they were deparaffinized and hydrated, and we performed antigenic recovery followed by incubation with monoclonal antibodies anti-TREM1 (Abcam, Cambridge, UK), anti-Def $\beta 2$ (Biorbyt, Cambridge, UK), anti-Dap12 (Abcam, Cambridge, UK), or anti-TLR2 (Millipore, Billerica, MA, USA) according to the manufacturer's instructions. The images were obtained using a Nikon E600 microscope and an Olympus Q-Color 1 digital camera and were quantified using ImageJ software.

\subsection{Statistical Analysis}

Statistical analyzes were performed using the GraphPad-Prism 8.0 statistical software. Comparisons between the two groups were performed using the Mann-Whitney nonparametric test, and analyses with more than two groups were performed using the 
Kruskal-Wallis or the Friedman test followed by Dunn's post test. ${ }^{*} p<0.05,{ }^{* *} p<0.01$, and ${ }^{* * *} p<0.001$ were considered statistically significant.

\section{Results}

\subsection{TREM-1 Signaling Pathway Is Significantly Up-Regulated in CL}

The transcriptome data (GSE55664 and GSE63931) of the human skin biopsies of the lesions caused by L. braziliensis were analyzed in order to identify key the pathways associated with CL. We evaluated the datasets using the "Core analysis" module of the IPA software to identify the canonical pathways related to the "Innate Immune response" during CL. The TREM-1 receptor signaling pathways were among the most up-regulated pathways in both datasets (ranked fourth in both datasets) (Figure 1A,D). To explore the TREM-1 pathway, volcano plots were constructed based on the whole transcriptome data genes, and the genes related to the TREM-1 pathway were identified (red dotsFigure 1B,E). For both datasets, 19 TREM-1 pathway genes were found to be significantly up-regulated. We further detailed the fold change values for each gene (Figure 1C,F), which showed a strong correspondence of gene expression between the two datasets. Together, these results indicate that the TREM-1 pathway is significantly activated in CL lesions.

A

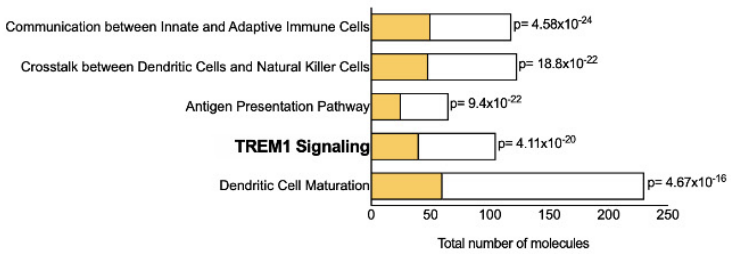

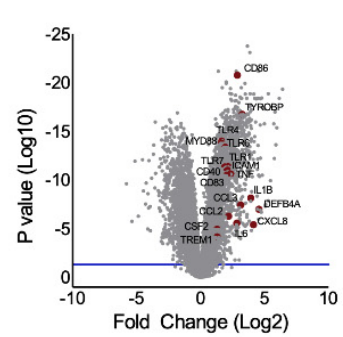

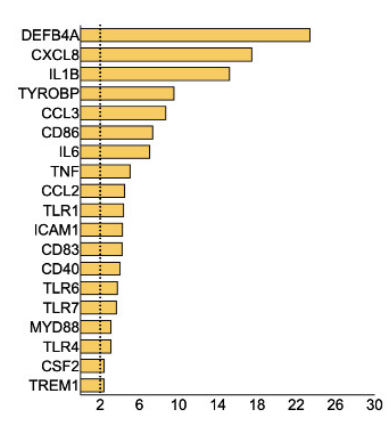

B

D

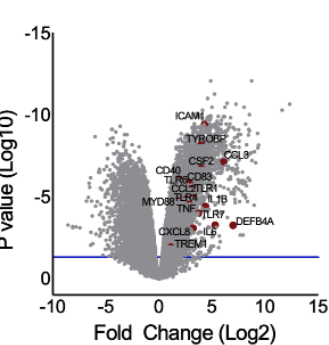

$\mathbf{F}$

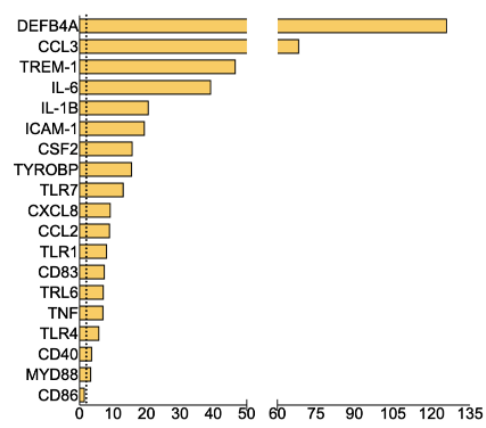

Figure 1. TREM-1 pathway is up-regulated in the lesions of CL patients. Canonical pathways identified in CL lesions by Ingenuity Pathway Analysis (IPA) software for the (A) GSE55664 and (B) GSE63931 datasets. Bars represent the number of genes in a pathway that are present in each data set found up-regulated (yellow) or down-regulated (blue). Volcano plots show gene expression of the entire (C) GSE55664 and (D) GSE63931 datasets, highlighting the genes from TREM-1 pathway. Fold change $>2$ (dashed line) of gene expression from TREM-1 pathway for the (E) GSE55664 and (F) GSE63931 datasets. 


\subsection{Expression Profile of Genes from the TREM-1 Pathway Distinguishes CL Samples from} Healthy Controls

Next, heatmaps were built to show the gene expression profile of the TREM-1 pathway for each sample, and hierarchical clustering analyses differentiated the control from the CL samples for both datasets (Figure 2A,B). We further performed Principal Component Analysis (PCA) based on data variances that confirmed differential expression of the TREM1 pathway between the controls and the CL samples (Figure 2C,D). These results confirm the differential regulation of the TREM-1 pathway in the biopsies from the CL lesions compared to the healthy skin samples.

A

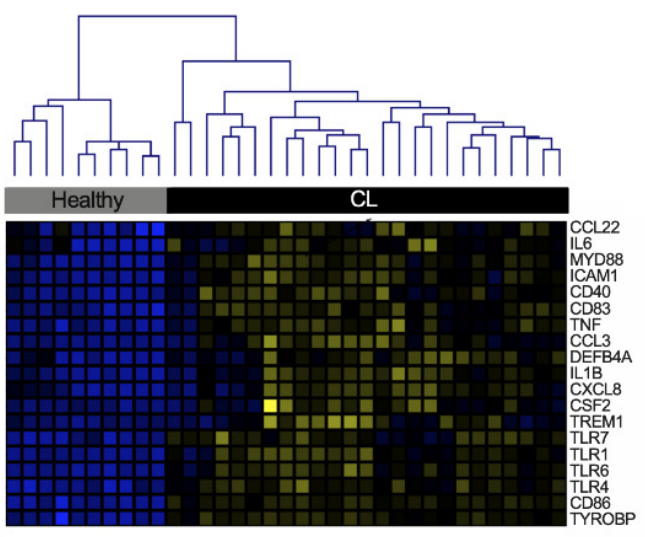

B

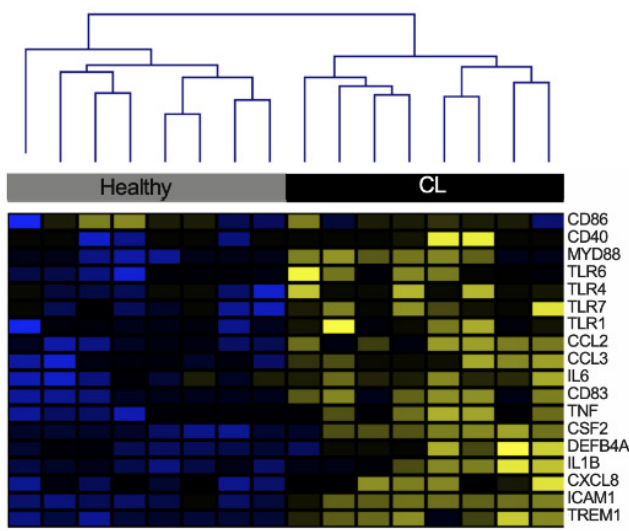

Intensity value $\left(\log _{2)}\right.$

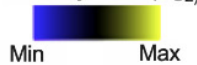

C

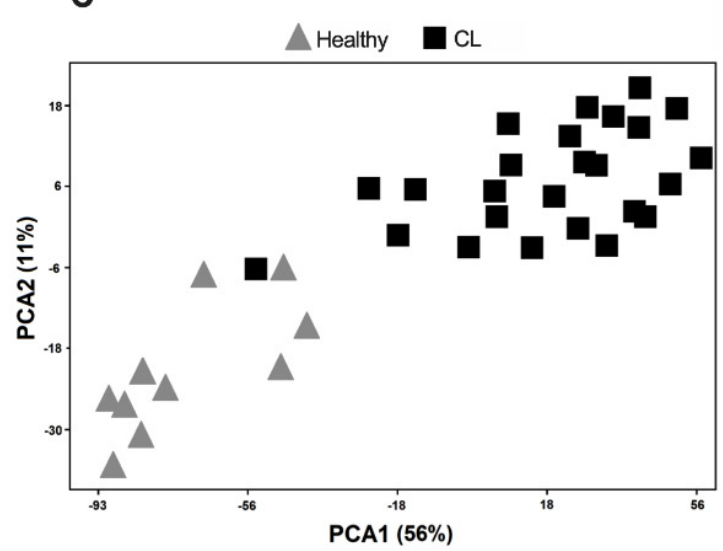

D

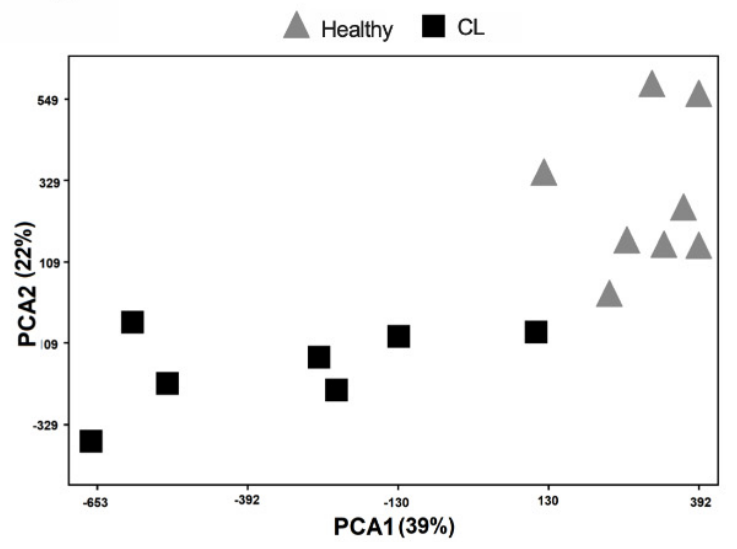

Figure 2. Differentially expressed genes of the TREM-1 pathway distinguished from the CL and control samples. Heatmaps of the Euclidean distance method realized by unsupervised hierarchical clustering of CL samples (black) and healthy controls (grey), showing the expression profile of 19 genes differentially expressed $(\mathbf{A}, \mathbf{B})$ and the Principal Component Analysis (C,D) of GSE55664 and GSE63931 datasets, respectively.

\subsection{Validation of Differentially Expressed Genes from TREM-1 Pathway in Active Lesions of CL}

Although the expression of TREM-1 and sTREM-1 (soluble form) has been demonstrated during the infection of different Leishmania species, the activation of the TREM-1 pathway in $C l$ lesions caused by $L b$ remains undetermined $[6,11-13,20]$. To validate the activation of the TREM-1 pathway in CL, some genes were selected based on their role within the pathway, such as receptors the TREM-1 and TLR2, the adaptor protein DAP12 (coded by TYROBP gene) and the product DEFB4A. We assessed new biopsies from patients with $C L$ caused by $L b$ to compare the expression of these genes with the healthy skin 
samples by means of RT-qPCR. TREM1, TLR2, TYROBP, and DEFB4A were significantly up-regulated in the CL lesions, considering a $p$ value $<0.05$ and a fold change greater than 2.0 (Figure 3A-D). These results confirm the findings from publicly available datasets and show that L. braziliensis infection enhances the expression of TREM- 1 and molecules associated with its activation.

A

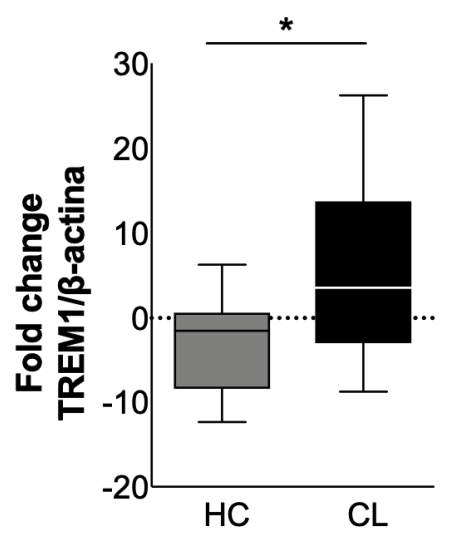

C

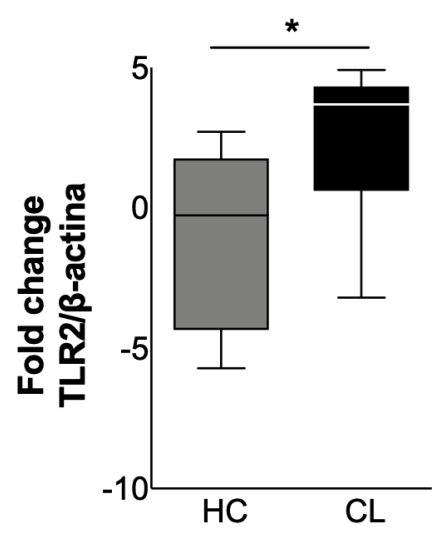

B

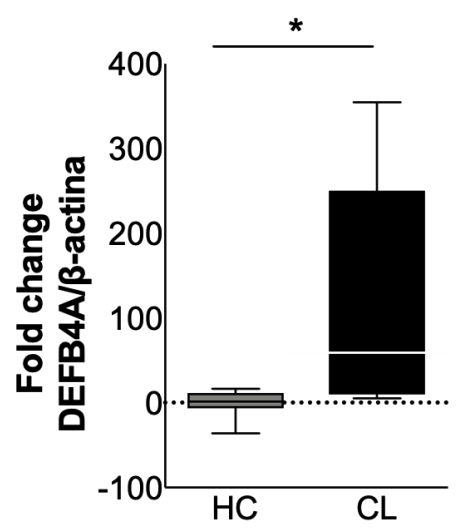

D

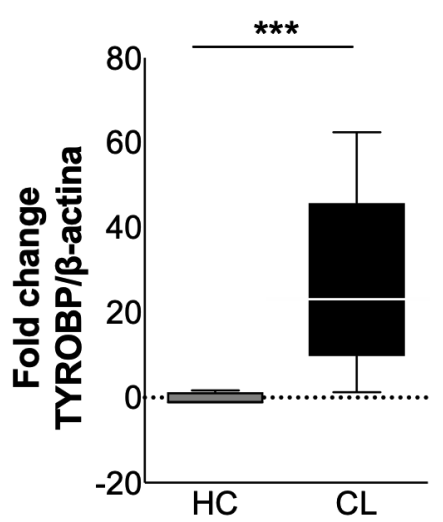

Figure 3. Increased expression of genes from the TREM-1 signaling pathway in CL lesions. Relative gene expression profile of (A) TREM-1 $(n=10),(\mathbf{B})$ DEFB4A $(n=7),(\mathbf{C})$ TLR2 $(n=9)$ and TYROBP $(n=6)$, and (D) by RT-qPCR. Data analyzed by the Mann-Whitney $\mathrm{t}$ test comparing CL biopsies (black bars) with healthy controls (grey bars). Bars represent the median \pm SEM and ${ }^{*} p \leq 0.05$, $* * * p \leq 0.001$. HC (healthy control).

\subsection{TREM-1 Protein Is Highly Expressed in the Epidermis of CL Lesions from Patients Infected} with L. braziliensis

To evaluate the presence of TREM-1 and other proteins associated with its pathway, we labeled these molecules in the tissue biopsies from the CL lesions with immunohistochemistry. Corroborating our gene expression results, this approach revealed higher TREM-1 expression in the epidermis as well as in the inflammatory infiltrate of the CL lesions when compared to healthy skin samples (Figure 4A). The quantification of the labeled area indicates that over $50 \%$ of the cells in the lesions were immunostained with TREM-1 (Figure 4B). We further quantified TREM-1 labelling between the epidermis and the inflammatory infiltrate using an automatized method. The results show 52 positive cells $/ \mathrm{mm}^{2}$ of the epidermis compared to 28 positive cells $/ \mathrm{mm}^{2}$ of the inflammatory infiltrate. This estimate suggests that proportionally, keratinocytes are expressing more TREM-1 than the inflammatory infiltrate, which could play a relevant role in CL lesions. Similar results were observed for TLR-2, DAP12, and DEFB4A, where increased protein 
labeling was shown in the inflammatory infiltrate of CL lesions compared to healthy skin (Figure 4A,B). These results confirm that the TREM-1 pathway is strongly induced in the skin affected by L. braziliensis infection and that it may have a key role in the exacerbated inflammation that characterizes this disease.

A
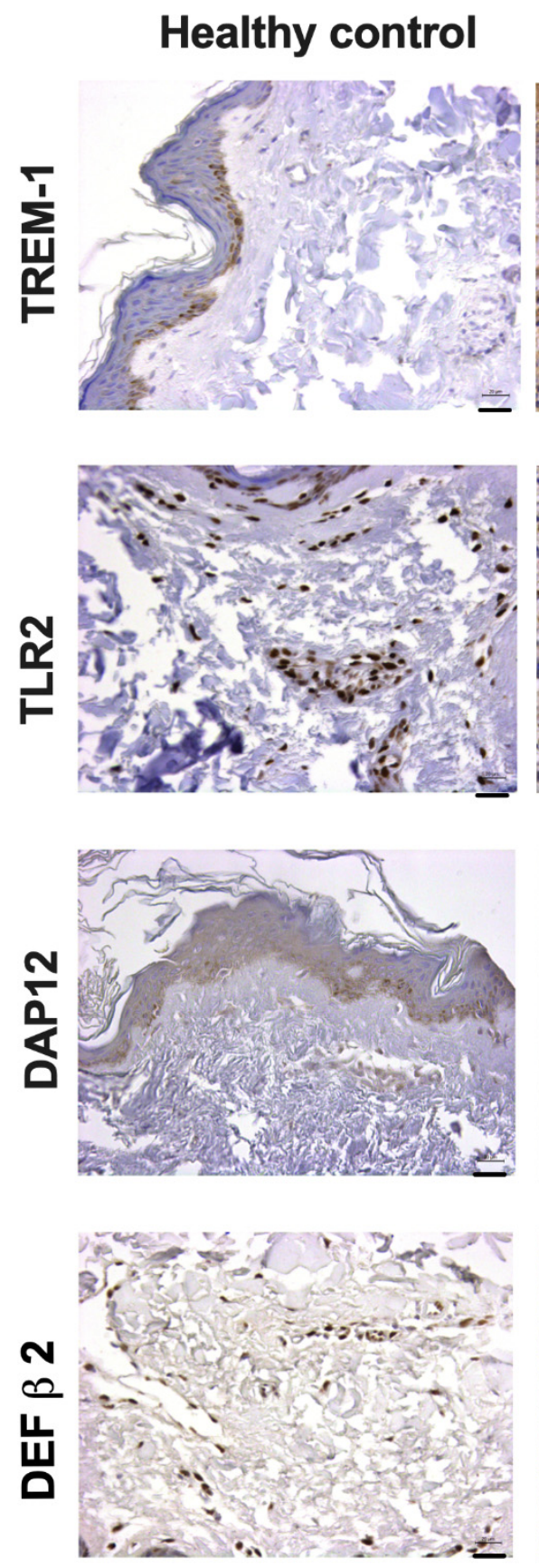

B
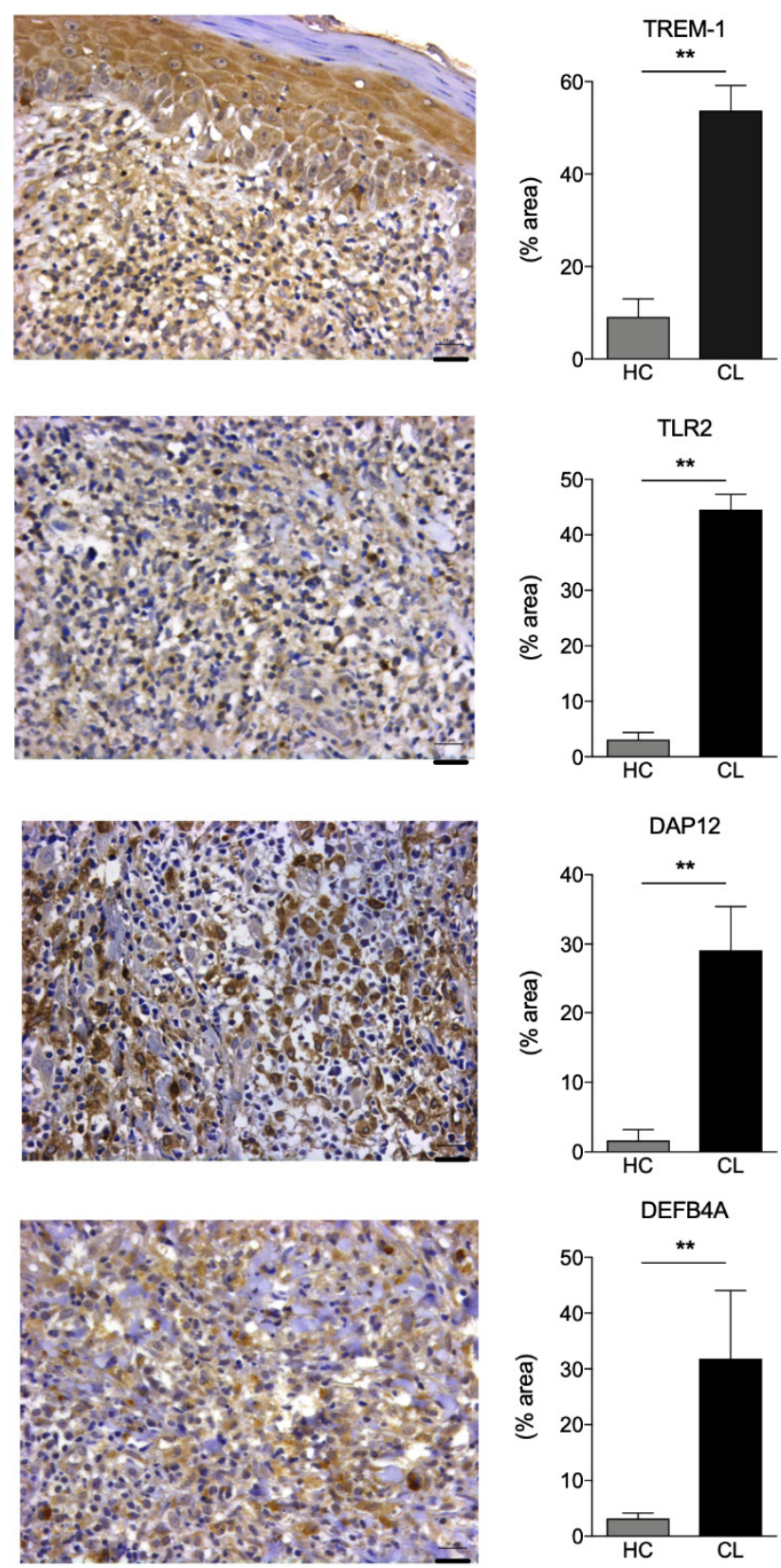

Figure 4. Increased protein levels of molecules from the TREM-1 pathway in biopsies from CL lesions caused by Lb infection. (A) Immunohistochemistry for TREM-1, TLR2, DAP12, and DEFB4A of lesions from CL patients. Long scale bars represent $20 \mathrm{~mm}$. Black rectangles designate region of 400 magnification. (B) Quantification of protein expression from TREM-1 pathway molecules by the Image J software. Representative images of immunohistochemistry label from biopsies of lesions caused by L. braziliensis compared to healthy skin samples $(n=6)$. $p$ value $<0.01^{* *}$ were considered significant. 
3.5. Gene Expression of TREM-1 Is Increased in Keratinocytes Exposed to L. braziliensis but Not to Mechanical Damage

Considering our findings on TREM-1 pathway activation in CL lesions, we next evaluated its expression and its main genes in human keratinocyte (HaCat) in vitro when exposed to different inflammatory conditions, such as L. braziliensis or scratch (mechani$\mathrm{cal} /$ physical damage to cells). Results obtained with this approach indicate that TREM-1 expression is only increased after L. braziliensis exposure (Figure 5A), and no difference is observed for scratch (Figure 5C). On the other hand, the expression of DEFB4A is increased for all of the tested conditions: L. braziliensis (Figure 5B), scratch (Figure 5D). These findings suggest that $L$. braziliensis infection increases the expression of TREM-1, whereas DEFB4A modulation seems to be an unspecific result of a pathogen (or its molecules) or cell damage.

A
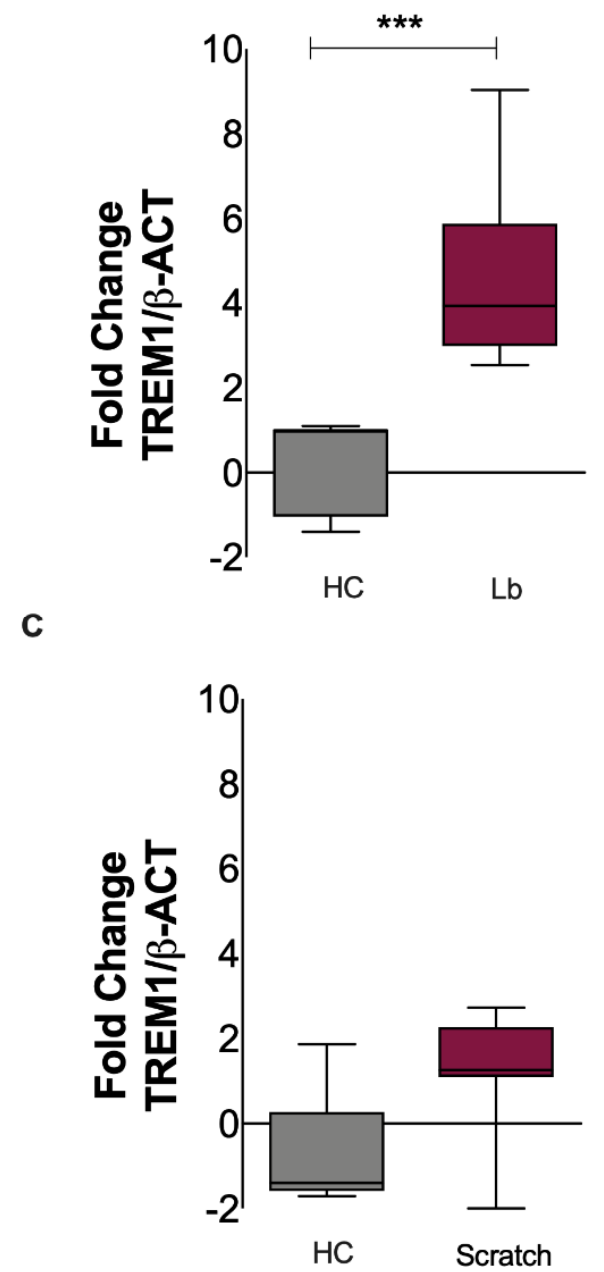

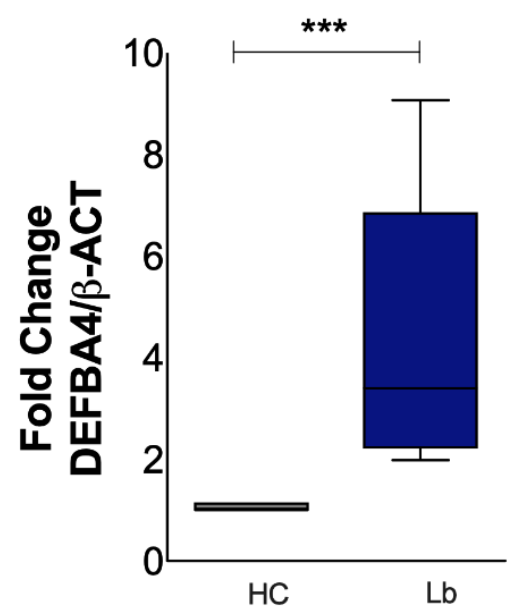

D

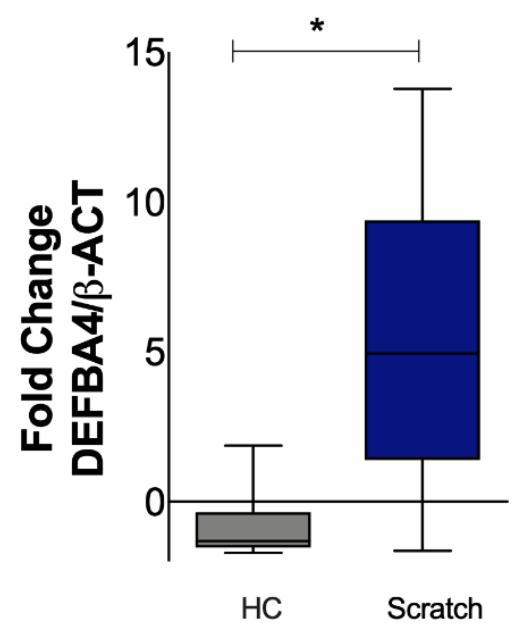

Figure 5. Differential TREM-1 and DEFB4A expression by human keratinocytes exposed to L. braziliensis or mechanical damage (scratch). Lineage human keratinocytes (HaCaT) were exposed to L. braziliensis at a 5:1 ratio (A,B) or scratch to mimic cell damage in the absence of a pathogen $(\mathbf{C}, \mathbf{D})$. After $4 \mathrm{~h}$, the relative expression of TREM-1 (red bars) and DEFB4A (blue bars) was analyzed by RT-qPCR and is represented as a simulated fold change compared to the unstimulated (grey bars) cultures. Bars represent median \pm standard error of two independent experiments performed in triplicate and quadruplicate each. The Mann-Whitney $t$ test considered significant $p$ values $<0.05^{*}$ and $<0.001 * * *$.

\subsection{Overexpression of TREM-1 Is Specific of CL and Is Not Observed in Other Non-Infectious Inflammatory Skin Diseases}

We next compared the expression profiles of TREM- 1 and DEFB4A found in L. braziliensis skin lesions with other inflammatory skin diseases in order to evaluate their specificity for CL. The GEO database was used to search for transcriptome datasets of non-infectious 
inflammatory skin diseases. We found two transcriptomes matching these criteria: one from psoriasis (GSE53431) and the other from systemic lupus erythematous (SLE) (GSE72535). The expression profile of DEFB4A was found to be significantly increased in all of the evaluated diseases (Figure 6). On the other hand, the expression of TREM-1 was significantly induced only in samples from patients with CL (GSE55664 and GSE63931). These data suggest that the expression of defensin DEFB4A is induced in inflammatory skin diseases regardless of their etiology, and TREM-1 expression appears to be specific for CL.

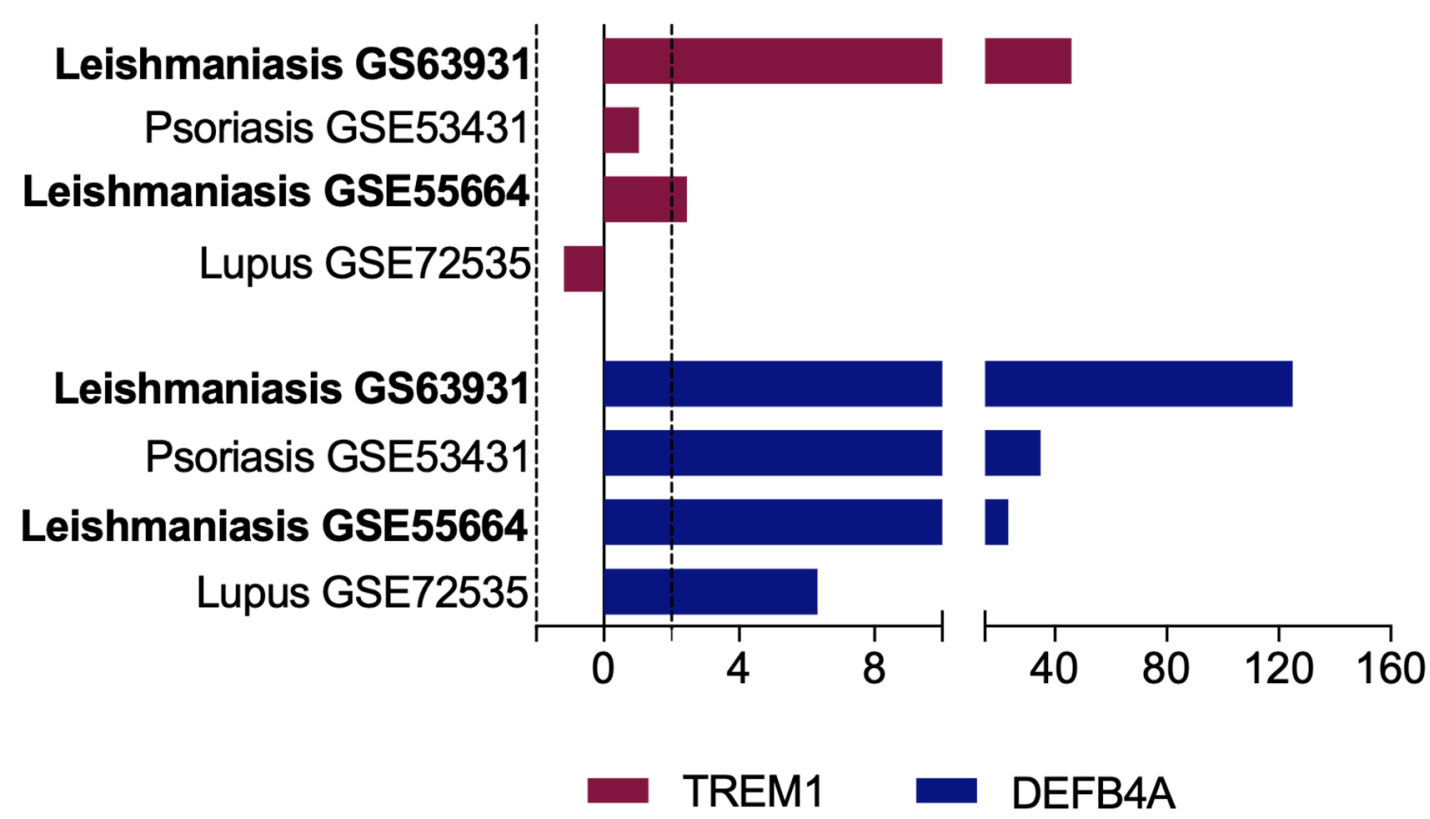

Figure 6. Activation of TREM-1 signaling is specific to skin lesions affected by L. braziliensis infection. Expression profile of TREM-1 (crimson bars) and DEFB4A (blue bars) in different inflammatory diseases of the skin analyzed from publicly transcriptome datasets for psoriasis (GSE53431), lupus (GSE72535), and leishmaniasis (GSE55664 and GSE63931). Fold change $>2$ (dashed line) of gene expression.

\section{Discussion}

TREM-1 has emerged as a key molecule during inflammatory diseases, promoting complex effects on cells of the immune system and exacerbating the host response $[7,8]$. Studies have explored the role of TREM- 1 in inducing inflammation in multiple contexts, such as sepsis [9,21], cancer [22], viral infections [23,24], and others non-infectious diseases $[25,26]$. However, the role of TREM- 1 signaling in innate immunity against parasite infections and its underlying mechanisms remain unknown $[13,27,28]$.

Here, we identified the TREM-1 pathway as an element of the innate immune response elicited in CL caused by L. braziliensis. The overexpression of TREM-1 and other molecules of this pathway, such as TYROBP, TLR2 and DEFB4A, were confirmed in new biopsies from CL patients.

Considering infectious diseases, the activation of the TREM-1 pathway has been more associated with disease severity than with infection control [6-8]. Studies have shown that influenza-infected Trem $1^{-1-}$ mice exhibit reduced morbidity but an equal capacity for viral clearance [6]. Similarly, the infection of Trem $1^{-/-}$mice with L. major leads to smaller inflammatory lesions with decreased neutrophilic cellular infiltrates in Trem $1^{-/-}$mice. However, the parasite counts did not differ between the groups [6]. In accordance with this data, another study reported that the TREM-1 signaling pathway is highly activated by L. major infection in susceptible mice [20]. In addition, studies show that the plasma levels of sTREM-1 are associated with the severity of malaria in children [26,29]. Regarding Schistosoma infection, some substances from Schistosoma eggs may inhibit the expression of TREM-1 on mouse macrophages. This mechanism can decrease the macrophage-mediated 
inflammatory response in infected hosts [28]. On the other hand, neutrophils infected by $L$. infantum enhance the transcription of TREM- 1 and molecules associated with its activation as well as the release of sTREM-1. Both were associated with clinical parameters of visceral leishmaniasis severity, suggesting that the release of sTREM-1 inhibits inflammation and favors disease development by impairing TREM-1 signaling [13].

Despite these findings in several infectious diseases, few studies have investigated the role of the TREM-1 in L. braziliensis infection, the main etiological agent of CL in Brazil. A previous report with peripheral blood mononuclear cells from healthy donors stimulated in vitro with L. braziliensis only found an activated TREM-1 pathway in cells from producers with high levels of IFN- $\gamma$ [11]. Moreover, a metanalysis from our group revealed correlations between TREM-1, miR193b, and miR-671 associated with a good response to treatment in human CL caused by L. braziliensis [12]. However, the activation of the pathway has remained unexplored.

In the present study, our immunohistochemical analysis revealed a significant increase in the expression of TREM- 1 and other proteins of its pathway in the biopsies of patients with CL. Similar results were found in lineage human keratinocytes (HaCats) exposed to $L$. braziliensis. However, unlike the exposure to Leishmania, the expression of TREM-1 mRNA in samples exposed to mechanical damage (scratch) did not show a significant increase of this molecule. These data suggest that the modulation of TREM-1 expression is strongly correlated with L. braziliensis infection.

Keratinocytes are the major structural component of the epidermis, but emerging evidence indicates their role in the immune response [30,31]. These cells express certain PRRs, including TLRs [32-35], which is an important pathway for Leishmania recognition by phagocytic cells and parasite internalization [36-38]. It has been shown that keratinocytes internalize L. major or L. infantum at low levels in vitro [14], but the exact mechanism by which keratinocytes recognize and phagocyte Leishmania is still unknown. Despite their inefficient phagocytosis of Leishmania, they robustly respond to parasite exposition [14], suggesting that surface molecules from Leishmania contribute to keratinocyte activation.

Several molecules are involved in the inflammatory processes associated with TREM-1 signaling, among them, Toll-like receptors, adaptor proteins and defensins. The activation of TREM-1 is associated with the DAP12 protein, which is encoded by the gene TYROBP and its ligand, which is still unknown. DAP12 couples to multiple receptors on the cell surface, and, when combined with TREM-1, contributes to the amplification of the inflammatory response by inducing the production of pro-inflammatory molecules $[10,39,40]$. Our data show that the expression of TYROBP mRNA and DAP12 protein are significantly up regulated in samples from CL biopsies, corroborating previously published studies.

The TREM- 1 amplified response is also mediated via Toll-like receptors (TLRs), mainly TLR2 and TLR4. In association with pathogen-associated molecular patterns (PAMPs) and damage-associated molecular patterns (DAMPs), TREM-1 induces the release of cytokines and chemokines through the NFKB pathway. In addition, this mechanism may also induce the release of the antimicrobial peptides present in granulocytes, mainly in mucosal and cutaneous immune responses, such as in defensins [40-42].

Defensins are a large family of antimicrobial peptides widely that are distributed in animals and plants. They are considered part of the innate immune response, where they contribute to the antimicrobial activities of cells, especially granulocytes, developing functions associated with the prevention and control of infections, autoimmune diseases, and cancer $[41,43,44]$. According to previously published studies, our findings show that after exposure to L. braziliensis, the expression of DEFB4A significantly increases at both the transcriptional and protein levels. In addition, defensin response appears to be non-specific since it was observed in mechanical damage (scratch) and also in non-infectious skin diseases (psoriasis and lupus).

We further evaluated the specificity of the TREM-1 observed in response to L. braziliensis infection in vitro through the analysis of public transcriptome datasets of non-infectious inflammatory skin diseases (psoriasis and lupus). In contrast to the CL findings, the 
modulation of TREM-1 was not observed in these non-infectious skin diseases. On the other hand, the expression of $D E F B 4 A$ is upregulated in all of the evaluated diseases as well as in response to the different stimulations we tested in vitro. These findings suggest that the TREM-1 response may be specifically related to the development of CL lesions and that defensin may be a product of tissue damage, regardless of the disease etiology. Therefore, this study provides evidence that human keratinocytes are able to strongly express TREM-1, which highlights the potential of non-immune cells in the inflammatory response against Leishmania.

Author Contributions: Conceptualization, S.N., M.R.A., Í.B.-S., R.L. and N.M.T.; methodology, S.N., M.R.A., Í.B.-S., R.L. and N.M.T.; software, S.N., M.R.A., Í.B.-S., R.L. and N.M.T.; validation, S.N., M.R.A., Í.B.-S., R.L. and N.M.T.; formal analysis, S.N., M.R.A., Í.B.-S., R.L., F.R.L., S.M.A., R.K., P.R.S.O., A.B., V.S.B., C.I.B. and N.M.T.; investigation, S.N., M.R.A., Í.B.-S., R.L. and N.M.T.; resources, C.I.B. and N.M.T.; data curation, S.N., M.R.A., ÍB.-S., R.L. and N.M.T.; writing-original draft preparation, S.N., M.R.A., Í.B.-S., R.L., F.R.L., S.M.A., R.K., P.R.S.O., A.B., V.S.B., C.I.B. and N.M.T.; writing-review and editing, S.N., M.R.A., Í.B.-S., R.L., F.R.L., S.M.A., R.K., P.R.S.O., A.B., V.S.B., C.I.B. and N.M.T.; visualization, S.N., M.R.A., Í.B.-S., R.L., F.R.L., R.K., P.R.S.O., V.S.B., C.I.B. and N.M.T.; supervision, N.M.T.; project administration, C.I.B. and N.M.T.; funding acquisition, C.I.B. and N.M.T. All authors have read and agreed to the published version of the manuscript.

Funding: This research was funded by FAPESB (Fundação de Amparo à Pesquisa do Estado na Bahia), grant number PET0004/2015 (Edital PROINTER), CAPES (Coordenação de Aperfeiçoamento de Pessoal de Nível Superior), financial code 001 and CNPq (Conselho Nacional de Desenvolvimento Científico e Tecnológico).

Institutional Review Board Statement: The study was conducted according to the guidelines of the Declaration of Helsinki and was approved by the Ethical Committee of the Gonçalo Moniz Institute-Salvador, Bahia, Brazil (protocol code 42928215.9.0000.0040.

Informed Consent Statement: All participants completed the Informed Consent Statement.

Data Availability Statement: Gene Expression Omnibus (GEO) (https:/ / www.ncbi.nlm.nih.gov/ geo/) - GSE55664 and GSE63931 (accessed on 10 May 2017); GSE53431 and GSE72535 (accessed on 4 December 2017).

Acknowledgments: The authors thank all patients who participated of this study and the professional group that recruited the participants. The authors thank Andrezza Kariny and Isabele Coelho for secretarial assistance. The authors thank Zaira Onofre from the RT-PCR facility-PDTIS (FIOCRUZ).

Conflicts of Interest: The authors declare no conflict of interest.

\section{References}

1. Kaye, P.; Scott, P. Leishmaniasis: Complexity at the host-pathogen interface. Nat. Rev. Microbiol. 2011, 9, 604-615. [CrossRef]

2. WHO Leishmaniasis. Available online: https://www.who.int/news-room/fact-sheets/detail/leishmaniasis (accessed on 5 January 2021).

3. Scott, P.; Novais, F.O. Cutaneous leishmaniasis: Immune responses in protection and pathogenesis. Nat. Rev. Immunol. 2016, 16, 581-592. [CrossRef]

4. Carvalho, L.P.; Passos, S.; Schriefer, A.; Carvalho, E.M. Protective and pathologic immune responses in human tegumentary leishmaniasis. Front. Immunol. 2012, 3, 301. [CrossRef] [PubMed]

5. Scorza, B.M.; Carvalho, E.M.; Wilson, M.E. Cutaneous Manifestations of Human and Murine Leishmaniasis. Int. J. Mol. Sci. 2017, 18, 1296. [CrossRef] [PubMed]

6. Weber, B.; Schuster, S.; Zysset, D.; Rihs, S.; Dickgreber, N.; Schürch, C.; Riether, C.; Siegrist, M.; Schneider, C.; Pawelski, H.; et al. TREM-1 deficiency can attenuate disease severity without affecting pathogen clearance. PLoS Pathog. 2014, 10, e1003900. [CrossRef] [PubMed]

7. Bouchon, A.; Dietrich, J.; Colonna, M. Cutting Edge: Inflammatory Responses Can Be Triggered by TREM-1, a Novel Receptor Expressed on Neutrophils and Monocytes. J. Immunol. 2000, 164, 4991-4995. [CrossRef]

8. Colonna, M. TREMs in the immune system and beyond. Nat. Rev. Immunol. 2003, 3, 445-453. [CrossRef] [PubMed]

9. Bouchon, A.; Facchetti, F.; Weigand, M.A.; Colonna, M. TREM-1 amplifies inflammation and is a crucial mediator of septic shock. Nature 2001, 410, 1103-1107. [CrossRef] 
10. Klesney-tait, J.; Turnbull, I.R.; Colonna, M. The TREM receptor family and signal integration. Nat. Immunol. 2006, 7, 1266-1273. [CrossRef]

11. Carneiro, M.W.; Fukutani, K.F.; Andrade, B.B.; Curvelo, R.P.; Cristal, J.R.; Carvalho, A.M.; Barral, A.; Van Weyenbergh, J.; Barral-Netto, M.; de Oliveira, C.I. Gene Expression Profile of High IFN- $\gamma$ Producers Stimulated with Leishmania braziliensis Identifies Genes Associated with Cutaneous Leishmaniasis. PLoS Negl. Trop. Dis. 2016, 10, e0005116. [CrossRef]

12. Nunes, S.; Silva, I.B.; Ampuero, M.R.; de Noronha, A.L.L.; de Souza, L.C.L.; Correia, T.C.; Khouri, R.; Boaventura, V.S.; Barral, A.; Ramos, P.I.P.; et al. Integrated analysis reveals that miR-193b, miR-671, and TREM-1 correlate with a good response to treatment of human Localized cutaneous leishmaniasis caused by Leishmania braziliensis. Front. Immunol. 2018, 9, 640. [CrossRef] [PubMed]

13. Bomfim, L.G.S.; Magalhães, L.S.; Santos-Filho, M.A.A.; Peres, N.T.A.; Corrêa, C.B.; Tanajura, D.M.; Silva, A.M.; Lipscomb, M.W.; Borges, V.M.; Jesus, A.R.; et al. Leishmania infantum Induces the Release of sTREM-1 in Visceral Leishmaniasis. Front. Microbiol. 2017, 8, 2265. [CrossRef] [PubMed]

14. Scorza, B.M.; Wacker, M.A.; Messingham, K.; Kim, P.; Klingelhutz, A.; Fairley, J.; Wilson, M.E. Differential Activation of Human Keratinocytes by Leishmania Species Causing Localized or Disseminated Disease. J. Invest. Dermatol. 2017, 137, $2149-2156$. [CrossRef] [PubMed]

15. Rogers, M.; Kropf, P.; Choi, B.S.; Dillon, R.; Podinovskaia, M.; Bates, P.; Müller, I. Proteophosophoglycans regurgitated by Leishmania-infected sand flies target the L-arginine metabolism of host macrophages to promote parasite survival. PLoS Pathog. 2009, 5, e1000555. [CrossRef]

16. Pivarcsi, A.; Kemény, L.; Dobozy, A. Innate immune functions of the keratinocytes: A review. Acta Microbiol. Immunol. Hung. 2004, 51, 303-310. [CrossRef]

17. Ehrchen, J.M.; Roebrock, K.; Foell, D.; Nippe, N.; Von Stebut, E.; Johannes, M.; Viemann, D.; Varga, G.; Mu, C.; Schuberth, J.; et al. Keratinocytes Determine Th1 Immunity during Early Experimental Leishmaniasis. PLoS Pathog. 2010, 6, e1000871. [CrossRef]

18. Novais, F.O.; Carvalho, L.P.; Passos, S.; Roos, D.S.; Carvalho, E.M.; Scott, P.; Beiting, D.P. Genomic Profiling of Human Leishmania braziliensis Lesions Identifies Transcriptional Modules Associated with Cutaneous Immunopathology. J. Investig. Dermatol. 2015, 135, 94-101. [CrossRef]

19. Oliveira, P.R.S.; Dessein, H.; Romano, A.; Cabantous, S.; De Brito, M.E.F.; Santoro, F.; Pitta, M.G.R.; Pereira, V.; Lain, C.; Rodrigues, V.; et al. IL2RA Genetic Variants Reduce IL-2-Dependent Responses and Aggravate Human Cutaneous Leishmaniasis. J. Immunol. 2015, 194, 2664-2672. [CrossRef]

20. Ulusan, Ö.; Mert, U.; Sadıqova, A.; Öztürk, S.; Caner, A. Identification of gene expression profiles in Leishmania major infection by integrated bioinformatics analyses. Acta Trop. 2020, 208, 105517. [CrossRef]

21. Maiese, A.; Bolino, G.; Mastracchio, A.; Frati, P.; Fineschi, V. An immunohistochemical study of the diagnostic value of TREM-1 as marker for fatal sepsis cases. Biotech. Histochem. 2019, 94, 159-166. [CrossRef]

22. Fontana, R.; Raccosta, L.; Rovati, L.; Steffensen, K.R.; Jakobsson, T.; Melloni, G.; Bandiera, A.; Bergamini, A.; Maggioni, D.; Doglioni, C.; et al. Nuclear receptor ligands induce TREM-1 expression on dendritic cells: Analysis of their role in tumors. Oncoimmunology 2019, 8, 1554967. [CrossRef]

23. Amrun, S.N.; Tan, J.J.L.; Rickett, N.Y.; Cox, J.A.; Lee, B.; Michael, J.G.; Solomon, T.; Perera, D.; Ooi, M.H.; Hiscox, J.A.; et al. TREM-1 activation is a potential key regulator in driving severe pathogenesis of enterovirus A71 infection. Sci. Rep. 2020, 10, 3810. [CrossRef]

24. Pedro, V.; Neto, S.; De Carvalho, J.C.S.; Pimentel, V.E. Prognostic value of sTREM-1 in COVID-19 patients: A biomarker for disease severity and mortality. medRxiv 2020. [CrossRef]

25. Tammaro, A.; Derive, M.; Gibot, S.; Leemans, J.C.; Florquin, S.; Dessing, M.C. TREM-1 and its potential ligands in non-infectious diseases: From biology to clinical perspectives. Pharmacol. Ther. 2017, 177, 81-95. [CrossRef]

26. Adukpo, S.; Gyan, B.A.; Ofori, M.F.; Dodoo, D.; Velavan, T.P.; Meyer, C.G. Triggering receptor expressed on myeloid cells 1 (TREM-1) and cytokine gene variants in complicated and uncomplicated malaria. Trop. Med. Int. Health 2016, 21, 1592-1601. [CrossRef] [PubMed]

27. Zhang, Y.; He, J.; Zheng, H.; Huang, S.; Lu, F. Association of TREM-1, IL-1 $\beta$, IL-33/ST2, and TLR Expressions with the Pathogenesis of Ocular Toxoplasmosis in Mouse Models on Different Genetic Backgrounds. Front. Microbiol. 2019, 10, 2264. [CrossRef] [PubMed]

28. Cheng, P.C.; Lin, C.N.; Chen, Y.J.; Chang, F.S.; Tsaihong, J.C.; Lee, K.M. Triggering receptor expressed on myeloid cells (TREM)-1 participates in Schistosoma mansoni inflammatory responses. Parasite Immunol. 2011, 33, 276-286. [CrossRef]

29. Erdman, L.K.; Dhabangi, A.; Musoke, C.; Conroy, A.L.; Hawkes, M.; Higgins, S.; Rajwans, N.; Wolofsky, K.T.; Streiner, D.L.; Liles, W.C.; et al. Combinations of host biomarkers predict mortality among Ugandan children with severe malaria: A retrospective case-control study. PLoS ONE 2011, 6, e17440. [CrossRef]

30. Matejuk, A. Skin Immunity. Arch. Immunol. Ther. Exp. 2017, 66, 45-54. [CrossRef] [PubMed]

31. Tsepkolenko, A.; Tsepkolenko, V.; Dash, S.; Mishra, A.; Bader, A.; Melerzanov, A.; Giri, S. The regenerative potential of skin and the immune system. Clin. Cosmet. Investig. Dermatol. 2019, 12, 519-532. [CrossRef] [PubMed]

32. Santiago-Téllez, A.; Castrillón-Rivera, L.E.; Palma-Ramos, A.; Bello-López, J.M.; Sainz-Espuñes, T.; Contreras-Paredes, A.; Luna-Herrera, J.; Castañeda-Sánchez, J.I. Keratinocyte infection by Actinomadura madurae triggers an inflammatory response. Trans. R. Soc. Trop. Med. Hyg. 2019, 113, 392-398. [CrossRef] 
33. Koike, S.; Yamasaki, K. Melanogenesis connection with innate immunity and toll-like receptors. Int. J. Mol. Sci. 2020, 21, 9769. [CrossRef]

34. Shimada-Omori, R.; Yamasaki, K.; Koike, S.; Yamauchi, T.; Aiba, S. TLR3 augments glucocorticoid-synthetic enzymes expression in epidermal keratinocytes; Implications of glucocorticoid metabolism in rosacea epidermis. J. Dermatol. Sci. 2020, 100, 58-66. [CrossRef]

35. Altonsy, M.O.; Kurwa, H.A.; Lauzon, G.J.; Amrein, M.; Gerber, A.N.; Almishri, W.; Mydlarski, P.R. Corynebacterium tuberculostearicum, a human skin colonizer, induces the canonical nuclear factor- $\mathrm{kB}$ inflammatory signaling pathway in human skin cells. Immunity, Inflamm. Dis. 2020, 8, 62-79. [CrossRef]

36. Sánchez Luquez, K.Y.; Zerpa, O.; Paz-Villarraga, C.A.; Fernández-Mestre, M. Genetic variability of molecules involved in the disease pathogenesis in Leishmania infection. Exp. Parasitol. 2020, 218, 108007. [CrossRef]

37. Regli, I.B.; Passelli, K.; Martínez-Salazar, B.; Amore, J.; Hurrell, B.P.; Müller, A.J.; Tacchini-Cottier, F. TLR7 Sensing by Neutrophils Is Critical for the Control of Cutaneous Leishmaniasis. Cell Rep. 2020, 31, 107746. [CrossRef] [PubMed]

38. Tavares, N.M.; Araújo-Santos, T.; Afonso, L.; Nogueira, P.M.; Lopes, U.G.; Soares, R.P.; Bozza, P.T.; Bandeira-Melo, C.; Borges, V.M.; Brodskyn, C. Understanding the Mechanisms Controlling Leishmania amazonensis Infection In Vitro: The Role of LTB4 Derived From Human Neutrophils. J. Infect. Dis. 2014, 210, 656-666. [CrossRef]

39. Tessarz, A.S.; Cerwenka, A. The TREM-1/DAP12 pathway. Immunol. Lett. 2008, 116, 111-116. [CrossRef] [PubMed]

40. Zeng, H.; Ornatowska, M.; Joo, M.S.; Sadikot, R.T. TREM-1 expression in macrophages is regulated at transcriptional level by NF-кB and PU.1. Eur. J. Immunol. 2007, 37, 2300-2308. [CrossRef]

41. Ganz, T. Defensins: Antimicrobial peptides of innate immunity. Nat. Rev. Immunol. 2003, 3, 710-720. [CrossRef] [PubMed]

42. Arts, R.J.W.; Joosten, L.A.B.; Van Der Meer, J.W.M.; Netea, M.G. TREM-1: Intracellular signaling pathways and interaction with pattern recognition receptors. J. Leukoc. Biol. 2013, 93, 209-215. [CrossRef] [PubMed]

43. Prado-Montes de Oca, E. Human $\beta$-defensin 1: A restless warrior against allergies, infections and cancer. Int. J. Biochem. Cell Biol. 2010, 42, 800-804. [CrossRef] [PubMed]

44. Wilmes, M.; Sahl, H.G. Defensin-based anti-infective strategies. Int. J. Med. Microbiol. 2014, 304, 93-99. [CrossRef] [PubMed] 